\title{
Correction to: Effects of clam dredging on benthic ecology of two cultivated northern quahog beds with different harvest histories and sediment grain sizes
}

\author{
Renee Mercaldo-Allen ${ }^{1} \cdot$ Shannon Meseck $^{1}$ • \\ Ronald Goldberg $^{1} \cdot$ Paul Clark $^{1}$ • Catherine Kuropat ${ }^{1}$. \\ Julie M. Rose ${ }^{1}$
}

Published online: 18 October 2017

(C) Springer International Publishing AG 2017

\section{Correction to: Aquacult Int (2017) 25:1971-1985 \\ https://doi.org/10.1007/s10499-017-0167-5}

The original version of this article unfortunately contained an error where the symbol for phi size was inadvertently omitted from the text.

The corrected version of the sentences are given below.

\begin{abstract}
Sentence found in lines 12-14 should be rewritten as: Main effects of lease (harvest history), dredging treatment (dredged versus not dredged plots), mean sediment phi size $(\boldsymbol{\Phi})$, and season (sampling date significantly affected benthic community structure.

Sentence found in lines 20-23 should be rewritten as: Our results suggest that harvest frequency and/or sediment $\boldsymbol{\Phi}$ size may explain differences in benthic assemblages between leased areas with different dredging histories, while dredging had no measurable effect on sediment chemistry.
\end{abstract}

The online version of the original article can be found at https://oi.org/10.1007/s10499-017-0167-5

Renee Mercaldo-Allen

renee.mercaldo-allen@noaa.gov

1 NOAA Fisheries, Northeast Fisheries Science Center, Milford Laboratory, 212 Rogers Avenue, Milford, CT 06460, USA 


\section{Sediment sampling, grain size, and chemistry}

Page 3, second paragraph, lines 5-7 should be rewritten as: Depth of each grab sample was measured in millimeters, and $50 \mathrm{~g}$ of sediment was subsampled for later $\boldsymbol{\Phi}$ size analysis.

Page 5, third paragraph, line 1 should be rewritten as: Sediment $\Phi$ size analysis was conducted at the laboratory.

Page 5, third paragraph, lines 6-7: Flux calculations were made using Flick's Law of Diffusion and correcting for differences in sediment $\boldsymbol{\Phi}$ size (Meseck et al. 2014).

\section{Results}

Page 6, first paragraph, lines 2-6 should be rewritten as: Repeated measures analysis of chemistry data found no difference in oxygen flux or hydrogen sulfide concentrations between newly dredged and not dredged plots at either the 8 - $(p=0.93, p=0.36)$ or 3 -year beds $(p=0.75,0.56)$. Mean phi size $(\boldsymbol{\Phi})$ differed significantly between leases and measured 1.4 (medium sand) for the 8 -year vs. 2.2 (fine sand) for the 3 -year beds $(\mathrm{p}<0.001)$. For this reason, mean $\Phi$ size was used as a covariate in the PERMANOVA model.

Page 6, second paragraph, lines 1-3 should be rewritten as: PERMANOVA identified significant main effects of lease (harvest history), dredging treatment (newly dredged versus not dredged plots within lease), mean phi size $(\mathbf{\Phi})$, and season (sampling date) on benthic community composition (Table 1).

\section{Discussion}

Page 8, subheading under discussion section, should be rewritten as: Sediment $\boldsymbol{\Phi}$ size

Page 8, first paragraph, lines 1-2 should be rewritten as: Sediment phi $(\boldsymbol{\Phi})$ size differed significantly between the two shellfish leases, despite their close geographic proximity.

Page 10, first paragraph, last three lines should be rewritten as: In order to account for sediment differences observed between the shellfish leases, $\boldsymbol{\Phi}$ size was used as a covariate in statistical analysis of harvesting effects. 